\title{
Diffraction-limited bispectrum speckle interferometry of the nuclear region of the Seyfert galaxy NGC 1068 in the $H$ and $K^{\prime}$ bands ${ }^{\star}$
}

\author{
G. Weigelt ${ }^{1}$, M. Wittkowski ${ }^{2}$, Y. Y. Balega ${ }^{3}$, T. Beckert ${ }^{1}$, W. J. Duschl ${ }^{4}$, K.-H. Hofmann ${ }^{1}$, \\ A. B. Men'shchikov ${ }^{5}$, and D. Schertl ${ }^{1}$ \\ 1 Max-Planck-Institut für Radioastronomie, Auf dem Hügel 69, 53121 Bonn, Germany \\ e-mail: weigelt@mpifr-bonn.mpg.de \\ 2 European Southern Observatory, Karl-Schwarzschild-Str. 2, 85748 Garching bei München, Germany \\ 3 Special Astrophysical Observatory, Nizhnij Arkhyz, Zelenchuk region, Karachai-Cherkesia 357147, Russia \\ ${ }^{4}$ Institut für Theoretische Astrophysik der Universität Heidelberg, Tiergartenstraße 15, 69121 Heidelberg, Germany \\ 5 Institute for Computational Astrophysics, Saint Mary's University, Halifax, NS B3H 3C3, Canada
}

Received 1 March 2004 / Accepted 4 June 2004

\begin{abstract}
We present near-infrared bispectrum speckle interferometry studies of the nuclear region of the Seyfert 2 galaxy NGC 1068. A diffraction-limited $K^{\prime}$-band image with 74 mas resolution and the first $H$-band image with 57 mas resolution were reconstructed from speckle interferograms obtained with the SAO $6 \mathrm{~m}$ telescope. The resolved structure consists of a compact core and an extended northern and south-eastern component. The compact core is resolved at all position angles and has a north-western, tail-shaped extension as well as a fainter, south-eastern extension. The $K^{\prime}$-band $F W H M$ diameter of this compact core is approximately $18 \times 39$ mas or $1.3 \times 2.8 \mathrm{pc}(F W H M$ of a single-component Gaussian fit; fit range $30-80 \%$ of the telescope cut-off frequency; the diameter errors are \pm 4 mas), and the position angle (PA) of the north-western extension is $-16 \pm 4^{\circ}$. If $40 \%$ of the flux from the compact $K^{\prime}$ core is emission from a point source and $60 \%$ from a Gaussian intensity distribution, then a slightly larger $F W H M$ of approximately $26 \times 58$ mas is obtained for the compact $K^{\prime}$ component. In the $H$ band, the $F W H M$ diameter of the compact core is approximately $18 \times 45$ mas ( \pm 4 mas), and the PA is $-18 \pm 4^{\circ}$. The extended northern component $\left(\mathrm{PA} \sim 0^{\circ}\right)$ has an elongated structure with a length of about 400 mas or $29 \mathrm{pc}$. The extended south-eastern component is fainter than the northern component. The $K^{\prime}$ - and $H$-band fluxes from the resolved compact core were measured to be $350 \pm 90$ mJy (i.e., $\left.K^{\prime} \sim 8.2^{\mathrm{m}}\right)$ and $70 \pm 20 \mathrm{mJy}\left(H \sim 10.4^{\mathrm{m}}\right)$, respectively. The PA of $-16 \pm 4^{\circ}$ of the compact $18 \times 39 \mathrm{mas}$ core is very similar to that of the western wall $\left(\mathrm{PA} \sim-15^{\circ}\right.$ ) of the bright region of the ionization cone. This suggests that the $H$ and $K^{\prime}$-band emission from the compact core is both thermal emission and scattered light from dust near the western wall of a low-density, conical cavity or from the innermost region of a parsec-scale dusty torus that is heated by the central source (the dust sublimation radius of NGC 1068 is approximately $0.1-1 \mathrm{pc}$ ). The northern extended 400 mas structure lies near the western wall of the ionization cone and coincides with the inner radio jet $\left(\mathrm{PA} \sim 11^{\circ}\right)$. The large distance from the core suggests that the $K^{\prime}$-band emission of the northern extended component is scattered light from the western cavity region and the radio jet region.
\end{abstract}

Key words. techniques: interferometric - galaxies: fundamental parameters - galaxies: nuclei - galaxies: photometry galaxies: Seyfert - galaxies: individual: NGC 1068

\section{Introduction}

NGC 1068 is one of the nearest and brightest Seyfert galaxies at a distance of $14.4 \mathrm{Mpc}$, corresponding to a linear scale of $72 \mathrm{pc} \mathrm{arcsec}^{-1}$ (Tully 1988; Bland-Hawthorn et al. 1997). Therefore, it is one of the best candidates for testing the predictions of unification schemes (Antonucci \& Miller 1985) of active galactic nuclei (AGN). The main ingredient of these models is a geometrically and optically thick torus

* Based on observations made with the $6 \mathrm{~m}$ BTA telescope, which is operated by the Special Astrophysical Observatory (SAO), Russia. surrounding the central continuum source. This torus is able to obscure the central source if the angle between its polar axis and the observer's viewing direction is large enough. Such a torus would collimate radiation from the central source and lead to the ionization cone, which is visible, for example, in the Hubble Space Telescope (HST) [O III] image of NGC 1068 (Macchetto et al. 1994). Theoretical studies of AGN tori were reported, for example, by Krolik \& Begelmann (1988), Pier \& Krolik (1992, 1993), Granato \& Danese (1994), Efstathiou \& Rowan-Robinson (1995), Efstathiou et al. (1995), Granato et al. (1997), Manske et al. (1998), Nenkova et al. (2002), 
Gratadour et al. (2003), and Galliano et al. (2003). Other geometries have also been discussed, such as an extended disk or torus consisting of several molecular clouds (e.g., Cameron et al. 1993; Nenkova et al. 2002; Vollmer et al. 2004), or a warped disk (Phinney 1989; Sanders et al. 1989; Pringle 1996, 1997).

The first high-resolution observations of NGC 1068 in the infrared were reported by McCarthy et al. (1982) and Chelli et al. (1987). Thatte et al. (1997) determined an upper limit of 30 mas for the diameter of the dominant $K$-band core in NGC 1068 and measured its $K$-band flux. Furthermore, they found, for the first time, that $94 \%$ of the $K$-band light in the central 1 " originates from a $\leq 30$ mas diameter source and that the remaining $6 \%$ of the light in the central $1^{\prime \prime}$ comes from stars. Rouan et al. (1998) reported the first $J$-, $H$-, and $K$-band Adaptive Optics (AO) images of NGC 1068, determined an upper limit of the $K$-band core diameter of $\sim 9 \mathrm{pc}$, found an elongated structure at $\mathrm{PA} \sim 102^{\circ}$ perpendicular to the inner radio jet, and resolved an elongated northern and southern structure with a length of $\sim 400$ mas. Our bispectrum speckle interferometry observations with the SAO $6 \mathrm{~m}$ telescope (Wittkowski et al. 1998) allowed the reconstruction of the first diffractionlimited $K$-band image with 76 mas resolution of the central $30 \pm 8$ mas $(\sim 2 \mathrm{pc})$ core of NGC 1068. The reconstructed image is elongated and has a north-western tail at PA $\sim-20^{\circ}$. Weinberger et al. (1999) obtained a $K$-band visibility function of NGC 1068 with the $10 \mathrm{~m}$ Keck telescope as well as, for the first time, an $H$-band visibility with the 200 inch Palomar telescope. The phase of the Fourier transform of NGC 1068 was found to be consistent with zero. The $K$-band visibilities obtained are in good agreement with the observations reported by Wittkowski et al. (1998). Marco \& Alloin (2000) carried out AO observations of NGC 1068 at $3.5 \mu \mathrm{m}$ (resolution $0.24^{\prime \prime}$ ) and $4.8 \mu \mathrm{m}$ with the ESO $3.6 \mathrm{~m}$ telescope. They found a central core with a radius of less than $8 \mathrm{pc}$ and a structure with a PA of approximately $100^{\circ}$, interpreted as the dusty molecular torus. Furthermore, they revealed extended emission in an approximately N-S direction coinciding with the $12.5 \mu \mathrm{m}$ emission found by Bock et al. (1998, 2000). Gratadour et al. (2003) obtained high angular resolution $K$-band spectroscopic observations of the nucleus of NGC 1068 using the 3-D spectrograph GriF coupled to the PUEO AO system. Rouan et al. (2004) reported the first diffraction-limited $K-, L-$, and $M$-band VLT/NACO AO observations of NGC 1068. The azimuthal average of the $K$-band image confirms that the core is resolved. The spectacular $L$-and $M$-band images show several new structures in the region of the radio jet, for example, a northern spiral arm-like structure, a southern tongue, and a set of parallel elongated nodules on both sides of the radio jet.

Using the VLBA, Gallimore et al. (1997) mapped the radio source S1 (Muxlow et al. 1996; Gallimore et al. 1996a), which is believed to be located at the position of the AGN, at $8.4 \mathrm{GHz}$ with an angular resolution of $\sim 1 \times 2$ mas. This source $\mathrm{S} 1$ has a size of $\sim 10 \times 30 \mathrm{mas}$, and its long axis $\left(\mathrm{PA} \sim 106^{\circ}\right)$ is approximately perpendicular to the inner radio jet. The radiation is either synchrotron or free-free emission and probably originates in a disk or torus. Furthermore, the $\mathrm{H}_{2} \mathrm{O}$ masers, observed within $\mathrm{S} 1$ along a line with $\mathrm{PA} \sim 135^{\circ}$ (Greenhill et al. 1996), may trace a warped disk or a torus.

Astrometric studies have shown that the position of the $2.2 \mu \mathrm{m}$ peak approximately coincides with the position of the 3.5 , the 4.8 , and the $12.4 \mu \mathrm{m}$ peak, as well as with the center of the HST polarization map and with the radio component S1 (Braatz et al. 1993; Capetti et al. 1995; Gallimore et al. 1996a,b,c; Thatte et al. 1997; Marco et al. 1997; Marco \& Alloin 2000; Bock et al. 1998, 2000; Kishimoto 1999; Galliano et al. 2003; Rouan et al. 2004). These observations suggest that the position of the IR peak and the radio component $S 1$ is the location of the central engine of the AGN and that the NIR and MIR core is emission from hot dust in the vicinity of the central engine.

In this paper we present a new diffraction-limited $K^{\prime}$-band bispectrum speckle interferometry image of NGC 1068, the first $H$-band image, as well as $H$ - and $K^{\prime}$-band photometry, and we discuss the resolved $H$ - and $K^{\prime}$-band structures.

\section{Observations, data reduction, and results}

\subsection{Observations and data reduction}

New speckle interferograms of NGC 1068 were obtained with the $6 \mathrm{~m}$ telescope of the Special Astrophysical Observatory (SAO) in Russia between 1997 and 2003. The 1997 speckle data were recorded using a $256 \times 256$ pixel NICMOS 3 camera, while from 1999 to 2003 we used a $512 \times 512$ pixel HAWAII Array camera. The observational parameters of the new 1997-2003 data, together with the 1996 data (Wittkowski et al. 1998), are listed in Table 1. For absolute $H$ - and $K$-band photometry we recorded calibration frames of three different photometric standard stars (Table 1). The visibilities of NGC 1068 were obtained using the speckle interferometry method (Labeyrie 1970). The images of NGC 1068 were reconstructed using the bispectrum speckle interferometry method (Weigelt 1977; Weigelt \& Wirnitzer 1983; Lohmann et al. 1983; Hofmann \& Weigelt 1986).

The following data processing steps were applied to the data sets:

- Calculation of the ensemble average power and bispectrum of each NGC 1068 and reference star data set. The bispectrum of each frame consisted of $\sim 37$ million elements (maximum length of bispectrum vectors: $u=45 \%$ of the telescope diffraction cut-off frequency $u_{\mathrm{DL}}, v=100 \%$ of $u_{\mathrm{DL}}$ ). The longest baselines used for the reconstructions had a length of exactly $6 \mathrm{~m}$.

- Compensation of the power and bispectrum noise bias (Pehlemann et al. 1992).

- Compensation of the speckle transfer function in the power and bispectrum.

- To overcome the influence of seeing variations, we alternately recorded calibrator and NGC 1068 data sets (16 calibrator and 16 object data sets in total).

- Recursive (Lohmann et al. 1983) reconstruction of the NGC 1068 images from their bispectra.

No postprocessing by image deconvolution methods was applied to the bispectrum speckle interferometry reconstructions 
Table 1. Observations. The columns list the following data set parameters: date, central wavelength $\lambda, F W H M$ bandwidth $\Delta \lambda$, pixel scale, fieldof-view (FOV I = FOV of recorded subframes, FOV II = FOV of the smaller subframes used for the calculation of the visibilities and images), number of recorded NGC 1068 frames, seeing, exposure time per frame, calibrator stars, and number of calibrator frames.

\begin{tabular}{|c|c|c|c|c|c|c|c|c|c|c|}
\hline Date & $\begin{array}{c}\lambda \\
(\mu \mathrm{m})\end{array}$ & $\begin{array}{c}\Delta \lambda \\
(\mu \mathrm{m})\end{array}$ & $\begin{array}{r}\text { Pix. scale } \\
\text { (mas/pixel) }\end{array}$ & $\begin{array}{r}\text { FOV I } \\
\left({ }^{\prime \prime}\right)\end{array}$ & $\begin{array}{r}\text { FOV II } \\
\left({ }^{\prime \prime}\right)\end{array}$ & $\begin{array}{l}\text { No. of } \\
\text { frames }\end{array}$ & $\begin{array}{l}\text { Seeing } \\
\left({ }^{\prime \prime}\right)\end{array}$ & $\begin{array}{l}\text { Exp. } \\
\text { (ms) }\end{array}$ & $\begin{array}{l}\text { Calibrator } \\
\text { name }\end{array}$ & $\begin{array}{l}\text { No. of } \\
\text { cal. frames }\end{array}$ \\
\hline 03 Oct. 1996 & 2.19 & 0.41 & 30.8 & 7.9 & 2.96 & 846 & 1.5 & 200 & HIP 12014 & 555 \\
\hline 23 Jan. 1997 & 2.19 & 0.41 & 30.8 & 7.9 & 2.96 & 1747 & 1.1 & 150 & HIP 12815 & 1748 \\
\hline 19 Oct. 1997 & 2.19 & 0.41 & 30.8 & 7.9 & 2.96 & 598 & 1.9 & 200 & HIP 13045 & 603 \\
\hline 22 Sep. 1999 & 2.16 & 0.32 & 26.4 & 5.1 & 2.53 & 1823 & 1.1 & 160 & HIP 12443 & 3209 \\
\hline 22 Sep. 1999 & 1.65 & 0.32 & 20.1 & 3.9 & 3.86 & 2232 & 0.9 & 120 & HIP 12598 & 1369 \\
\hline 26 Sep. 1999 & 2.16 & 0.32 & 26.4 & 3.4 & 2.53 & 5528 & 1.0 & 90 & HIP 12598 & 4909 \\
\hline 27 Sep. 1999 & 2.16 & 0.32 & 26.4 & 3.4 & 2.53 & 5605 & 0.9 & 90 & HIP 12598 & 1982 \\
\hline 14 Oct. 2003 & 2.12 & 0.21 & 28.7 & 7.3 & 2.75 & 4906 & 2.2 & 240,320 & SAO 130081 & 3151 \\
\hline
\end{tabular}

$H$ - and $K^{\prime}$-band absolute photometry of NGC 1068 was performed by observing three different photometric standard stars: 22 Sep. 1999 : Gl 105.5 (40 frames); 19 Sep. 2002: Gl 105.5, HD 22686, and G77-31 (40 frames/star).

since the reconstructed images are not degraded by any instrumental or atmospheric point spread function (the resolved yellow/red structures of the $K^{\prime}$ and $H$ images in Fig. 4 are in the range of $1-100 \%$ and $4-100 \%$ of the peak intensities, respectively). The resolved $18 \times 39$ mas object shows that it is even possible to reconstruct diffraction-limited images of structures which are slightly smaller than the size of the Airy disk.

\section{2. $H$ - and $K^{\prime}$-band visibilities and autocorrelations}

Figures 1 and 2 show all reconstructed two-dimensional $K^{\prime}$ and $H$-band visibility functions of NGC 1068 obtained at four epochs between 1997 and 2003 as well as the $K$-band visibilities reconstructed from the 1996 data (Wittkowski et al. 1998), cuts through the two-dimensional visibilities along the major and minor axes, and cuts through the model fits of twodimensional elliptical Gaussian brightness distributions. This single-component fit and a two-component fit (a Gaussian intensity distribution plus a point source) are discussed below in more detail. In the following text, in both the two-dimensional visibilities and the images, north is up, and east is to the left. The visibilities obtained from the three September 1999 data sets were averaged. All obtained visibilities show similar, elongated shapes. The elongated shape of the 1996-2003 visibilities is also consistent with the elongated shape $\left(\mathrm{PA} \sim-20^{\circ}\right.$ ) of the true image of NGC 1068 shown in Wittkowski et al. (1998). The low-frequency peak of all visibility functions at spatial frequencies of up to $\sim 2 \operatorname{arcsec}^{-1}$ is caused by the host galaxy and an additional extended component discussed below in more detail.

The shapes of all visibility functions show that the dominant compact core of NGC 1068 is elongated. We have derived the size of this resolved compact core of NGC 1068 by fitting a single-component elliptical Gaussian brightness distribution (a two-component model fit is discussed below) to the data in the range of 30 to $80 \%$ of the telescope cut-off frequency. The $K^{\prime}$ and $H$-band telescope cut-off frequencies are at spatial frequencies of approximately 13 and $17 \operatorname{arcsec}^{-1}$, respectively. This fit range excludes both the low-frequency peak (host galaxy and extended component) and the less accurate, highest spatial frequencies.

Table 2 shows the resulting model parameters of the compact core for each data set as well as the average of all 1999 (good seeing) and 2003 (bad seeing) $K^{\prime}$-band results. This average was calculated by weighting the data sets proportionally to their numbers of frames. The difference between the 1999 and 2003 values is also listed in order to illustrate the good agreement in spite of the large differences in seeing and numbers of frames. We obtained an average $K^{\prime}$ diameter ( $F W H M$, Gaussian fit) of $18.4 \times 39.4$ mas and a PA of $-16^{\circ}$ (for the long axis in image space; errors are discussed below). In the following text we call this resolved compact core, which has a similar but not identical size in the $H$ band, simply the $18 \times 39$ mas object or component.

The $F W H M H$-band diameters of the minor and major axes of the compact component are 18.4 and 44.7 mas, respectively, and the PA is $-18^{\circ}$ (Table 2 ).

To derive the total error of these values, we split the data sets into a larger number of smaller data sets and studied how the sizes depend on the compensation of the speckle interferometry and bispectrum transfer function, the compensation of noise-bias terms in the power and bispectrum (Pehlemann et al. 1992), and the recursive derivation of the Fourier phase of the object from the bispectrum. The result of this investigation is that the total error of the derived $K^{\prime}$ - and $H$-band sizes is \pm 4 mas, and the error of the position angles is $\pm 4^{\circ}$.

Furthermore, the visibilities allow us to derive an upper limit for the flux from an additional, unresolved component which may exist within the $18 \times 39$ mas object. Such a point source would contribute a constant component to the total visibility function. The total flux of each component of the visibility can be derived from the zero-frequency value of its visibility. The zero-frequency value of the compact component is $\sim 0.82$ (see Fig. 1) in the 1999 visibility function derived from subframes with a FOV of 2.5 arcsec. We can only use the 1999 data for this analysis since in 2003 seeing varied very much during data recording, and therefore, the visibilities at low spatial frequencies have large calibration errors (Christou et al. 1985). The $1999 K^{\prime}$-band visibilities show 

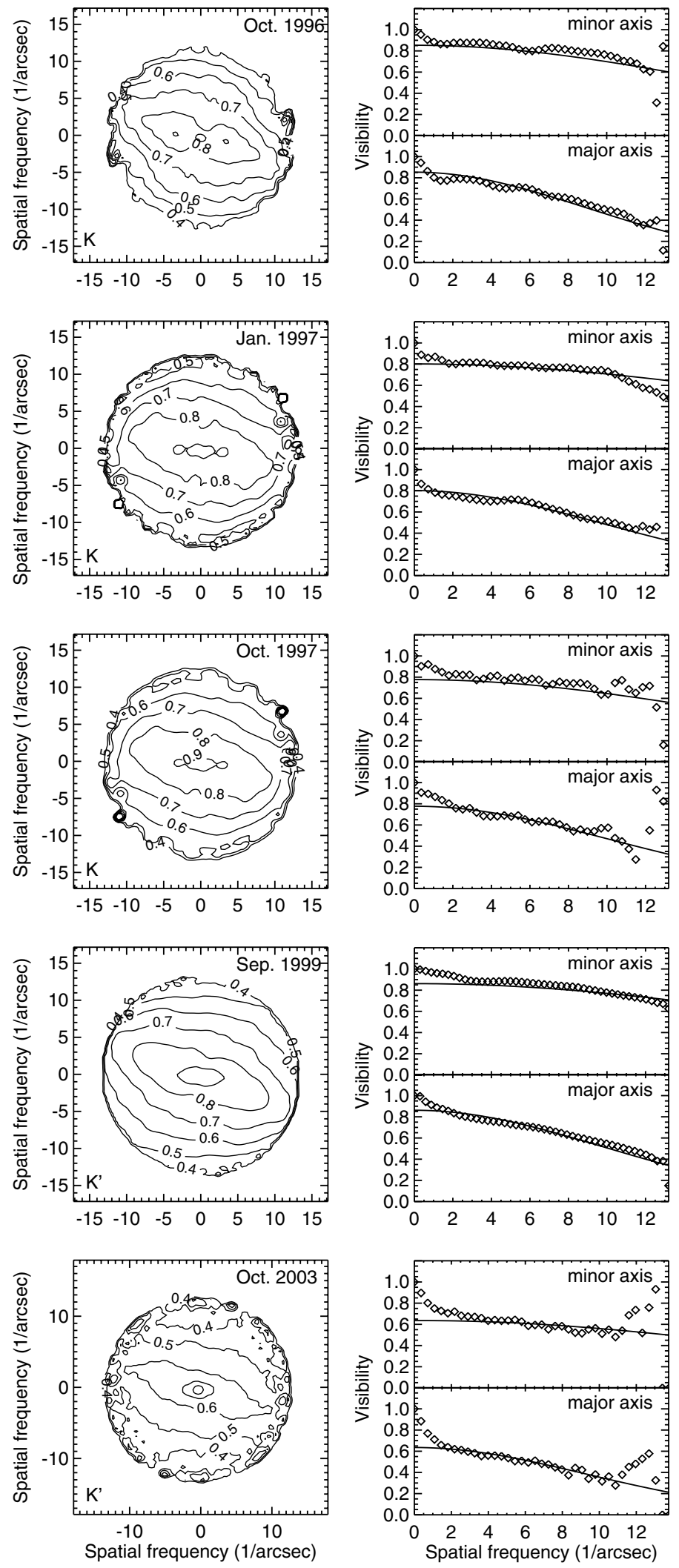

that the low-frequency visibility peak at spatial frequencies below $2 \operatorname{arcsec}^{-1}$ has a relative flux contribution of $\sim 18 \%$. This low-frequency visibility peak is caused by the host galaxy and the extended 400 mas structure (discussed below), which together contribute $\sim 18 \%$ of the $K^{\prime}$ flux in the $2.5 \times 2.5^{\prime \prime}$ FOV.
Fig. 1. Left: $K$ - or $K^{\prime}$-band visibilities of NGC 1068 observed in 1996, 1997, 1999, and 2003. Right: cuts through their major and minor axes. North is at the top and east to the left in the corresponding image. The contours are spaced from 40 to $90 \%$ in 6 steps. The diamonds are observations, and the solid lines are cuts through fits of two-dimensional Gaussian brightness distributions (fit range 30 to $80 \%$ of the telescope cut-off frequency).

Furthermore, the 1999 visibility value of $\sim 0.38$ at the diffraction cut-off frequency (minor axis) shows that an additional, constant visibility component would have a relative flux contribution of less than 0.38 of the total flux within a 2.5 arcsec FOV or a relative flux less than $\sim 0.38 / 0.82=0.46( \pm 0.05)$ of 

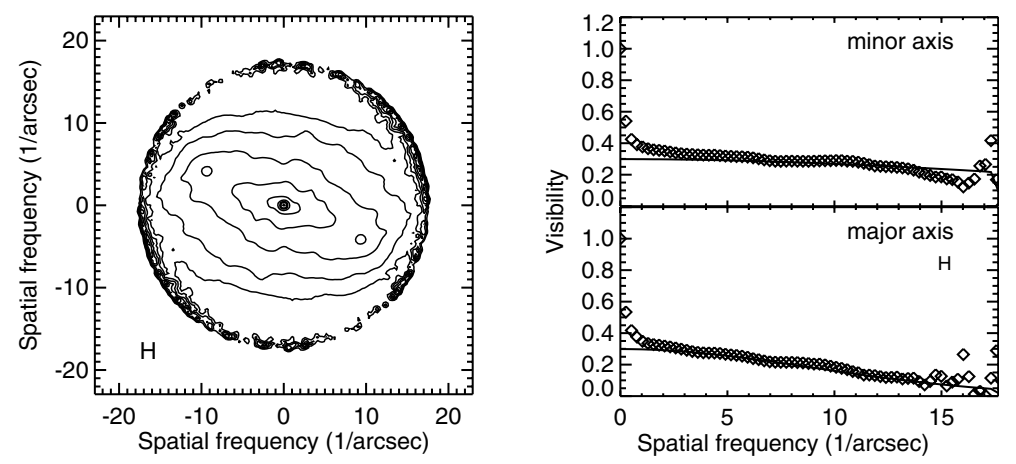

Fig. 2. Two-dimensional $H$-band visibilities of NGC 1068 and cuts through their major and minor axes. The contours are spaced from 10 to $90 \%$ in 18 linear steps. Diamonds are observations, solid lines are fits of a two-dimensional Gaussian brightness distribution.

Table 2. Model fit parameters of the two-dimensional visibilities using a single elliptical Gaussian intensity distribution. The columns list major-to-minor axes ratio, the minor and major axes $(F W H M)$, the PA of the major axis in image space, and the azimuthally averaged $F W H M$ diameter (Gaussian fit). The two lines at the bottom give the average of the 1999 and $2003 K^{\prime}$-band data (the average was calculated by weighting the data sets proportionally to the number of frames) and the difference between the 1999 (good seeing) and 2003 (bad seeing) data.

\begin{tabular}{lrrrrr}
\hline \hline Date (band) & $\begin{array}{r}\text { Axes } \\
\text { ratio }\end{array}$ & $\begin{array}{r}\text { Minor } \\
(\text { mas })\end{array}$ & $\begin{array}{r}\text { Major } \\
(\text { mas })\end{array}$ & $\begin{array}{r}\text { PA } \\
\left({ }^{\circ}\right)\end{array}$ & $\begin{array}{r}\text { Diam. } \\
(\text { mas })\end{array}$ \\
\hline Oct. 1996 $(K)$ & 2.1 & 18.8 & 40.0 & -21 & 26.5 \\
Jan. 1997 $(K)$ & 2.0 & 20.0 & 40.3 & -12 & 27.5 \\
Oct. 1997 $(K)$ & 1.7 & 24.2 & 39.9 & -26 & 30.6 \\
Sep. 1999 $\left(K^{\prime}\right)$ & 2.1 & 17.9 & 38.5 & -16 & 25.3 \\
Sep. 1999 $(H)$ & 2.4 & 18.4 & 44.7 & -18 & 27.3 \\
Oct. 2003 $\left(K^{\prime}\right)$ & 2.1 & 19.6 & 41.9 & -15 & 27.7 \\
\hline Average $\left(K^{\prime}\right)$ & 2.1 & 18.4 & 39.4 & -16 & 26.0 \\
99/03 diff. $\left(K^{\prime}\right)$ & 0 & 1.7 & 3.4 & 1 & 2.4 \\
\hline
\end{tabular}

the flux of the compact component. Therefore, our visibilities are consistent with an unresolved substructure within the compact component if its flux contribution is less than $46 \pm 5 \%$ of the flux of the $18 \times 39$ mas $K^{\prime}$-band component.

Finally, we studied how the size of the compact $K^{\prime}$-band component (1999 plus 2003 data) changes if we fit a twocomponent model to the visibilities. The two-component model consists of a two-dimensional elliptical Gaussian intensity contributing $60 \%$ of the flux plus a point source contributing $40 \%$. With this model we derived a larger $F W H M$ diameter of $26 \times 58$ mas for the compact component.

Figure 3 shows the $K^{\prime}$ - and $H$-band autocorrelations of NGC 1068. In both panels the compact autocorrelation of the $18 \times 39$ mas core and extended structures are visible.

\subsection{Diffraction-limited $\mathrm{K}^{\prime}$ - and $\mathrm{H}$-band images}

Figure 4 presents our reconstructed diffraction-limited $K^{\prime}$-band image (resolution 74 mas) derived from the 1999 data and the first diffraction-limited $H$-band image of NGC 1068 with a resolution of 57 mas. In these images, the compact $18 \times 39$ mas object, which caused the elongated shape of the visibilities, can clearly be recognized. The $K^{\prime}$ - and $H$-band images of the compact object show that it is asymmetric and has a north-western, tail-shaped extension at $\mathrm{PA} \sim-16^{\circ}$ as well as a weaker, southeastern extension (the compact structure is yellow in the $K^{\prime}$ and red in the $H$ image, and it is also visible in the contour plots).

Furthermore, a northern structure with a length of approximately 400 mas $\left(\mathrm{PA} \sim 0^{\circ}\right)$ as well as a south-eastern extended component is visible in the $K^{\prime}$-band image. In addition to the $18 \times 39$ mas core and the more extended components, the $K^{\prime}$-band image clearly shows the first diffraction ring around the compact component. The diffraction ring is inhomogeneous because of noise.

The northern and south-eastern extended components are in good agreement with the north-south features resolved by Rouan et al. (1998) (3.6 m telescope AO image with 120 mas resolution; PA of the north-south feature $\sim 4^{\circ}$ ), and by Marco $\&$ Alloin (2000) ( $L$ - and $M$-band AO images). The visibilities, autocorrelations, and $H$ - and $K^{\prime}$-band images of the compact $18 \times 39$ mas object are in good agreement with the $K$-band visibilities and image shown in Wittkowski et al. (1998), with $H$ - and $K$-band visibilities and autocorrelations reported by Weinberger et al. (1999), and with the diffraction-limited $K$-band VLT/NACO AO observations in Rouan et al. (2004).

The PA of $-16^{\circ}$ of the compact $18 \times 39$ mas core is very similar to the position angle of the western wall $\left(\mathrm{PA} \sim-15^{\circ}\right.$; Capetti et al. 1995) of the bright region of the ionization cone. This suggests that the $H$ - and $K^{\prime}$-band emission from the compact object is both thermal emission and scattered light from dust near the western wall of a low-density, conical cavity within the ionization cone or from the innermost region of a parsec-scale dusty torus that is heated by the central source. Dust emission from the corresponding eastern region of the cavity is not visible, possibly due to an asymmetric dust distribution. Such asymmetry is also observed in the MIR (Bock et al. 1998, 2000).

The northern extended 400 mas $K$-band structure $\left(\mathrm{PA} \sim 0 \pm 10^{\circ}\right.$ ) lies not exactly at but near the western wall of the ionization cone $\left(\mathrm{PA} \sim-15^{\circ}\right)$ and coincides with the inner radio jet $\left(\mathrm{PA} \sim 11^{\circ}\right)$. The brightness of this northern component is only $\sim 2 \%$ of the peak brightness of the core. In Fig. 4 (bottom) this northern component appears to end at the radio component $\mathrm{C}$, where the direction of the jet changes from $\mathrm{PA} \sim 11^{\circ}$ to $\sim 30^{\circ}$. The northern borderline of the northern component is approximately perpendicular to the outer radio jet. The PA of the south-eastern extended component is similar to that of the radio component $\mathrm{S} 2$ relative to $\mathrm{S} 1$. The large distance of most parts of the northern component from the core 

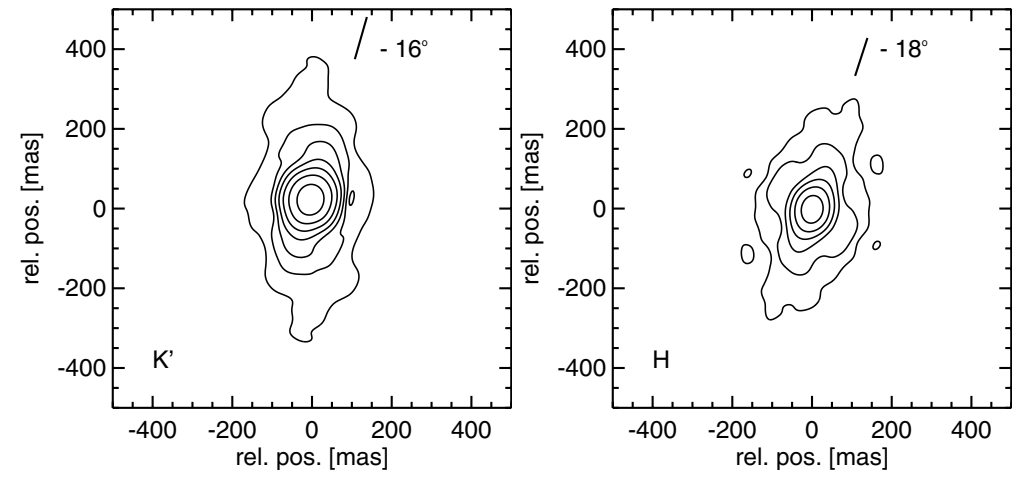

Fig. 3. Left: $K^{\prime}$-band autocorrelation of NGC 1068 derived from the 1999 data. The contours are plotted at levels of 1 , $2,4,8,16,32$, and $64 \%$ of the peak intensity. Right: $H$-band autocorrelation of NGC 1068. The contour levels are at 2,4 , $8,16,32$, and $64 \%$ of the peak intensity. suggests that the $K^{\prime}$-band emission of the northern extended component is scattered light from the western cavity region and from the radio jet region. In the $H$-band image, these extended structures are not visible, probably because the dynamic range of the $H$-band image is much lower than that of the $K^{\prime}$-band image.

\section{4. $\mathrm{K}^{\prime}$ - and $\mathrm{H}$-band absolute photometry}

We calibrated the $K^{\prime}$ - and $H$-band fluxes of the various structures by comparing the integral intensities of the NGC 1068 data with observations of several photometric standard stars (Table 1). Integrated $K^{\prime}$-band fluxes of $585 \mathrm{mJy}$ and $480 \mathrm{mJy}$ were obtained for the FOV of $5.0^{\prime \prime} \times 5.0^{\prime \prime}$ and $2.5^{\prime \prime} \times 2.5^{\prime \prime}$, respectively (22 Sep. 1999 data), while integrated $H$-band fluxes of $234 \mathrm{mJy}$ and $191 \mathrm{mJy}$ were obtained for the FOV of $3.9^{\prime \prime} \times 3.9^{\prime \prime}$ and $2.5^{\prime \prime} \times 2.5^{\prime \prime}$, respectively (22 Sep. 1999).

The flux ratio of the compact component and the extended structure (i.e., the host galaxy and the northern and southern extended components) can be derived from the visibility function at spatial frequency zero, as discussed above. We can only use the 1999 data for this analysis, since in 2003 seeing varied very much during data recording, and therefore, the visibilities at low spatial frequencies have large calibration errors (Chistou et al. 1985). We determined the $K^{\prime}$ - and $H$-band flux contribution (Sep. 1999) of the $18 \times 39$ mas component to be $350 \pm 90 \mathrm{mJy}$ (i.e., $K^{\prime} \sim 8.2^{\mathrm{m}} \pm 0.3^{\mathrm{m}}$ ) and $70 \pm 20 \mathrm{mJy}$ $\left(H \sim 10.4^{\mathrm{m}} \pm 0.3^{\mathrm{m}}\right)$, respectively. These magnitudes show that the compact $18 \times 39$ mas component is very red $\left(H-K^{\prime} \sim 2.2^{\mathrm{m}}\right)$.

In Feb. 1997 Rouan et al. (1998) observed the fainter core magnitudes $K=9.3^{\mathrm{m}}$ (flux of the undeconvolved AO image within a circular $0.2^{\prime \prime}$ diameter aperture) and $H=13.1^{\mathrm{m}}$ (flux within a $0.2^{\prime \prime}$ diameter aperture in an image in which the extended component was removed by subtracting two exponential disks with characteristic radii of 3.1 and $0.26^{\prime \prime}$ from the AO image). One possible explanation for the different core magnitudes is that two very different methods were used for subtracting the extended component. Another possibility is the reported variability of the nucleus (Glass 1997).

In our speckle observations, the determination of the core brightness is a two-step process. In the first step, the integrated flux within a small aperture is determined (absolute photometry) as discussed above. We estimate the error of the determined integrated $K^{\prime}$-band flux of $585 \mathrm{mJy}$ in the FOV of $5.0^{\prime \prime} \times 5.0^{\prime \prime}$ to be less than $20 \%$ since we used three different photometric standard stars, and the observations were carried out during good photometric conditions. This value is similar to the flux of $739 \mathrm{mJy}\left(K=7.31^{\mathrm{m}}\right)$ reported by Scoville (1988) for the slightly longer wavelength of $2.20 \mu \mathrm{m}$. The second step is the determination of the relative flux fraction of the $18 \times 39$ mas component. As discussed above, the 1999 visibilities show that this flux fraction is $82 \%$ of the total flux in a FOV of $2.5^{\prime \prime} \times 2.5^{\prime \prime}$ (this flux fraction can be derived from the visibility at spatial frequency zero since the total flux within a given area is equal to $\int i(x) \mathrm{d} x=\int i(x) \exp [-2 \pi i x 0] \mathrm{d} x=$ Fourier transform of $i(x)$ at spatial frequency zero, if $i(x)$ is the intensity distribution). In Fig. 1 we show the visibilities obtained during different seeing conditions. This comparison of all the visibilities observed is quite interesting. The very good 1999 data $\left(K^{\prime}\right.$-band seeing $\left.0.9-1.0^{\prime \prime}\right)$ yield a flux fraction of the compact core of 0.82 , and the two shorter 1997 data sets taken during worse seeing conditions yield similar values of 0.80 in $1 / 1997$ (1.1" seeing) and 0.79 in 10/1997 (1.9" seeing). These values obtained at quite different seeing conditions illustrate the robustness of the method and that it is very likely that the error of the flux fraction 0.82 is smaller than \pm 0.05 . Only the extremely bad 2003 data (2.2" $K^{\prime}$-band seeing!) yield the much smaller flux fraction of 0.61 .

Finally, we also measured the brightness of the core of NGC 1068 in archival HST NICMOS images. From the $F 205 W$-filter image n4as01bj (taken on 11 Oct. 1997) we derived the magnitudes $7.78,8.06$, and $8.24^{\mathrm{m}}$ within circular apertures with radii of 2,1 , and $0.5^{\prime \prime}$, respectively. If we take into account the average flux from the galaxy background (measured in an annulus with inner and outer radii of 1 and $2^{\prime \prime}$ ), we obtain the background-subtracted core magnitudes (=magnitudes of the $18 \times 39$ mas component) of $8.27^{\mathrm{m}} \pm 0.3^{\mathrm{m}}$ for the filter $F 205 \mathrm{~W}$ and $7.76^{\mathrm{m}} \pm 0.3^{\mathrm{m}}$ for the filter $F 222 M$ (image n4hk03010;21 Feb. 1998). With the same backgroundsubtraction method, we obtained the $H$-band flux of $10.1^{\mathrm{m}} \pm$ $0.3^{\mathrm{m}}$ for the compact core (filter $F 160 \mathrm{~W}$; NICMOS image n4hk02010; date 21 Feb. 1998.

As discussed above, our visibilities would also be consistent with an additional, unresolved substructure within the $18 \times 39$ mas component if its relative flux is less than $46 \pm 5 \%$ of the compact component. This means that the $K^{\prime}$ flux of such an unresolved component would be less than $\sim 161 \pm 43 \mathrm{mJy}$. 

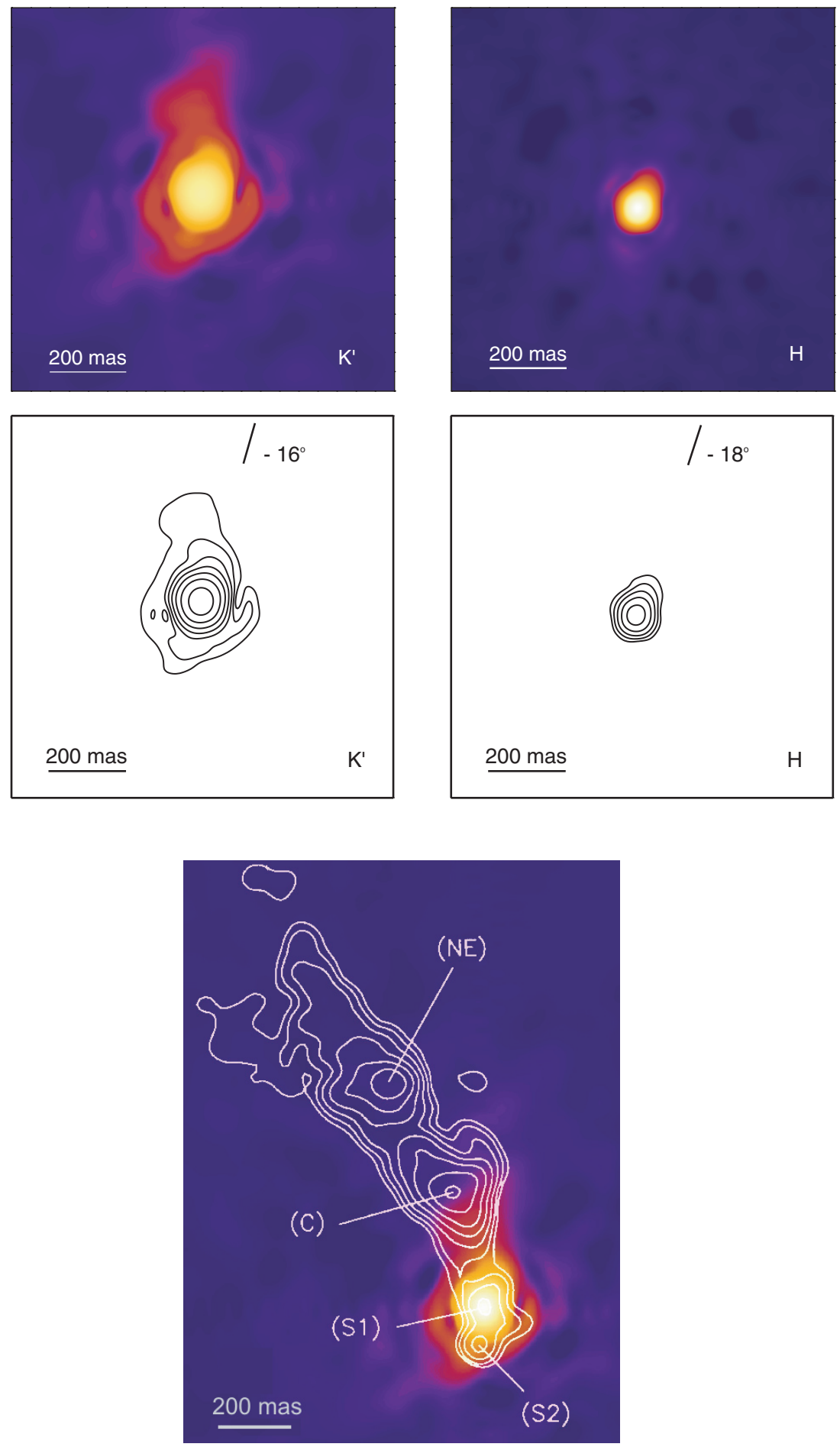

Fig. 4. Left, top and middle: Diffraction-limited $K^{\prime}$-band image of NGC 1068 reconstructed by bispectrum speckle interferometry. The image shows the compact core (yellow) with its tail-shaped, north-western extension at PA of approximately $-16^{\circ}$ as well as the northern and southeastern extended components (red). In addition to these structures, the first diffraction ring around the compact component is visible. The contour levels are $1,2,4,8,16,32$, and $64 \%$ of the peak intensity. North is up, and east is to the left. Right, top and middle: Diffraction-limited $H$-band image, which also shows the tail-shaped, north-western extension. The contour levels are 4, 8, 16, 32, and $64 \%$ of the peak intensity. Bottom: MERLIN $5 \mathrm{GHz}$ contour map (Gallimore et al. 1996b) superposed on our $K^{\prime}$-band image. The center of the radio component $\mathrm{S} 1$ coincides with the center of the $K^{\prime}$-band peak (see Sect. 1). The PA of $-16^{\circ}$ of the $18 \times 39$ mas $K^{\prime}$-band core is very similar to that of the western wall $\left(\mathrm{PA} \sim-15^{\circ}\right)$ of the bright region of the ionization cone. The northern extended 400 mas structure (red) aligns with the direction of the inner radio jet $(\mathrm{S} 1-\mathrm{C})$. The northern borderline of the northern component is almost perpendicular to the radio jet direction between $\mathrm{C}$ and NE. The PA of the south-eastern extended component is similar to the PA of S1-S2. 
From the $H$-band visibility, we derived that the flux of an unresolved $H$-band component would be less than $6 \pm 4 \mathrm{mJy}$.

The determination of the flux of the extended 400 mas component is difficult since the intensity distribution of the host galaxy is not known in the inner 400 mas. Therefore, only a very rough range of $30-230 \mathrm{mJy}$ can be derived for the $K^{\prime}$-band flux of the extended 400 mas component. The peak brightness of the extended component is approximately $2 \%$ of that of the compact component.

\section{Discussion and interpretation}

\subsection{Optically thin emission from hot dust}

Emission in the $K^{\prime}$ band may be interpreted quite naturally as scattered and direct thermal radiation of hot dust near its sublimation temperature $(\sim 1000-1500 \mathrm{~K})$, located at the inner edge of the dusty torus or in the polar cone between BLR and NLR. Dust grain temperatures can be estimated from the radiative equilibrium in an optically thin medium. The innermost regions of the torus are directly exposed to the radiation from the nucleus, and thus, even if the bulk of the torus is optically thick, our simple estimate will give a reliable value for the hottest temperatures of the torus. However, the derived dust temperatures critically depend on optical properties of dust grains in NGC 1068, which are not well known. Indications of grainsize distributions in AGN favoring large grains are reported by Maiolino et al. (2001) and are also discussed with respect to the nucleus of NGC 1068 (Galliano et al. 2003).

With different assumptions on the chemical composition and optical constants, the sublimation radius, at which dust reaches a temperature of $1500 \mathrm{~K}$, is estimated by Cameron et al. (1993) to be $0.14 \mathrm{pc}$ for a central luminosity of $1.5 \times 10^{11} L_{\odot}$. Thatte et al. (1997) follow the estimate of Barvainis (1987), which, for the same luminosity, results in a radius of $0.3 \mathrm{pc}$. At the other extreme Dopita et al. (1998) extrapolate a result of Puget et al. (1985) and find a radius of $1.2 \mathrm{pc}$.

In addition to the unknown dust properties, the luminosity of the central source is also uncertain; for example Bock et al. (2000) and Marco \& Alloin (2000) consider values greater than $2.5 \times 10^{12} L_{\odot}$ derived from a discussion of MIR and NIR color temperatures of observed, extended emission. The corresponding sublimation radius following Barvainis (1987) with $T_{\text {sub }}=1500 \mathrm{~K}$ is then $1.3 \mathrm{pc}$. The measured size of the $18 \times 39$ mas core, when interpreted as a dust free cavity with a size determined by the dust sublimation radius, agrees with the estimate by Barvainis (1987) for luminosities of about $10^{12} L_{\odot}$. The major axis of the resolved core points to the western wall of the ionization cone, and the extension in this direction is expected to be larger than the sublimation diameter.

The theoretically expected decline of the dust temperature with radius, $T \propto r^{-0.36 \ldots-0.44}$ (Barvainis 1987; Dopita et al. 1998), when only the central engine is responsible for the dust heating, implies that the extended 400 mas ( 29 pc) component cannot be explained by radiation of optically thin dust. However, it is very likely that a fraction of the $K^{\prime}$-band flux from the compact resolved core (radius $\sim 0.7-1.4 \mathrm{pc}$ ) is thermal emission from hot dust.

\subsection{Radiation from an optically thick torus}

The above example of optically thin thermal dust emission is certainly an oversimplification when applied to NGC 1068. For a quantitative analysis of the $K^{\prime}$-band flux emerging from the optically thick torus, accurate radiative transfer modeling is necessary. A number of models have been published for NGC 1068 (Pier \& Krolik 1992, 1993; Granato \& Danese 1994; Efstathiou \& Rowan-Robinson 1995; Efstathiou et al. 1995; Granato et al. 1997; Manske et al. 1998; Nenkova et al. 2002; Galliano et al. 2003). All these models try to fit the SED of Rieke \& Low (1975) with a $K$-band flux of $0.3 \pm 0.1 \mathrm{Jy}$.

A compact torus with high optical depth was studied by Pier \& Krolik $(1992,1993)$. The model assumes additional cold dust $(T \sim 130 \mathrm{~K})$ in the polar direction to explain the MIR to FIR flux and electrons in the ionization cone, which scatters $K$-band light into our line of sight. The inner radius of their best-fit model corresponds to an effective black body temperature of $800 \mathrm{~K}$ or $0.5 \mathrm{pc}$ for our adopted distance. Efstathiou et al. (1995) introduced in their extended torus an additional dust component located within the ionization cone to reach the required $K$-band flux. An extended torus with a different vertical stratification (Granato \& Danese 1994; Granato et al. 1997) provides a reasonable fit to the SED of Rieke \& Low (1975). The model has a small inner radius of $0.13 \mathrm{pc}$ (re-scaled to $D=14.4 \mathrm{Mpc}$ ) and a constant midplane density, so that most of the dust is located far from the nucleus.

The flux from the $18 \times 39$ mas object in our observations can, in agreement with torus models by Pier \& Krolik (1993), Efstathiou et al. (1995), and Granato et al. (1997), be attributed to the hot innermost torus region or near-nuclear dust in the ionization cone, while the more extended, northern 400 mas component would correspond to colder dust and electrons in the NLR scattering nuclear radiation into our line of sight.

Torus models which can explain the observed infrared SED by emission solely from the torus (i.e., without dust within the ionization cone) were presented by Granato \& Danese (1994). In a recent discussion Galliano et al. (2003) pointed out that various models with different combinations of parameters are consistent with the nuclear SED. The larger $K^{\prime}$ - and $H$-band fluxes reported in this paper may challenge some of these torus models. A simulated $2.2 \mu \mathrm{m}$ image reported by Granato et al. (1997) shows an elongated shape along the direction with low absorption. The scale of the image is set by the inner radius of the torus model (i.e., the model images are equal or somewhat larger than the dust sublimation radius). As discussed above, our images of the compact core also have a size which is similar to the dust sublimation radius. More detailed images of the brightness distribution on scales between 20 and 100 mas are required to further distinguish between different classes of models.

Manske et al. (1998) explored the consequences of an anisotropic central source and found that strong anisotropy can increase the NIR flux. They also noted the strong dependence of the radiation shortward of $5 \mu \mathrm{m}$ on the torus opening and viewing angle.

It was noted early on by Krolik \& Begelmann (1988) that dust in a geometrically thick torus will only survive in cool 
clumps and that clumpiness might be important in understanding the IR spectrum. The existence of clouds with high optical depth is supported by dynamical models (Vollmer et al. 2004; Beckert \& Duschl 2004) of a thick and clumpy torus. Nenkova et al. (2002) investigated clumpy tori in more detail and developed a statistical treatment of the radiative transfer in a clumpy environment. The broad-band nature of the IR spectrum of AGN is a consequence of dusty clouds being effectively heated both near and far from the central source due to clumpiness. Since the NIR emission probes the innermost region, fluctuations in the distribution of clumps will affect the $K$-band flux and allow deviations from the calculated mean.

\subsection{Radiation from stars}

The contribution of late-type stars to the flux in the central $1^{\prime \prime}$ was estimated by Thatte et al. (1997) from CO-band head features. It was found that $94 \%$ of the $K$-band light in the central $1^{\prime \prime}$ originates from a $\leq 30$ mas diameter compact core and that the remaining $6 \%$ of the light in the central 1 " comes from stars. From stellar synthesis they found that the corresponding stellar cluster is less than $1.6 \mathrm{Gyr}$ old, and the bolometric luminosity of the cluster within $1^{\prime \prime}$ is at least $2.7 \times 10^{9} L_{\odot}$.

\subsection{Scattered radiation}

The observed UV and optical continuum radiation of the NLR is interpreted as Thomson scattering of radiation of the central source by free electrons of the NLR (Miller et al. 1991). The electron cloud is distributed along the radio jet, has a size of about $1-2^{\prime \prime}$, and scatters $1.5 \%$ of the flux of the central continuum source into our line of sight. As the Thomsonscattering cross section is independent of wavelength, Pier \& Krolik (1993) assumed that NIR radiation, too, is scattered into our line of sight. Since our extended $K^{\prime}$-band component is traced up to $29 \mathrm{pc}$ where NLR clouds are located, it is likely that a fraction of its flux is the radiation scattered by electrons. Since the component appears to be aligned with the western wall of the cone and the inner radio jet, it may partly be the radiation scattered by dust grains and electrons.

\subsection{Emission from the central engine}

As dust extinction is usually lower at NIR wavelengths compared to optical wavelengths, there is a possibility that a certain fraction of the flux of the central source reaches us directly without being strongly absorbed or scattered. If the distribution of the obscuring material is more complex than a smooth optically and geometrically thick torus, this may be even more likely. A torus consisting of a number of dusty clouds will have a lower mean optical depth than a smooth dust distribution of equal mass. The same is true for other geometries, for example, a clumpy disk (Cameron et al. 1993) or a warped disk (e.g. Pringle 1997).

A non-negligible contribution at $2 \mu \mathrm{m}$ may come from an optically thick, geometrically thin standard accretion disk surrounding the central engine. Assuming a black hole mass of
NGC 1068 of $1.5 \times 10^{7} M_{\odot}$ (Greenhill et al. 1996), a mass accretion rate of $10^{-1} M_{\odot} / \mathrm{yr}$, and an inclination angle of $60^{\circ}$, the flux emerging from the accretion disk in the $K$ band is $\sim 60 \mathrm{mJy}$ (not corrected for dust extinction). Such a disk is on the verge of self-gravity (Duschl et al. 2000; Huré 2002), consistent with the sub-Keplerian rotation velocities observed by Greenhill et al. (1996).

Another possibility was discussed by Wittkowski et al. (1998): the spectrum of radio component $\mathrm{S} 1$ is inverted and has a spectral index of 0.3. A $F_{v} \propto v^{1 / 3}$ spectrum has also been observed in our Galaxy's center (Beckert et al. 1996) and M 81 (Reuter \& Lesch 1996). It can be explained by synchrotron emission of quasi monoenergetic relativistic electrons (Beckert \& Duschl 1997), and for higher electron energies in AGN the synchrotron spectrum may reach up to NIR wavelengths. With a mean electron energy of $\sim 2.7 \mathrm{GeV}$, a flux of $300 \mathrm{mJy}$ at $2 \mu \mathrm{m}$ from an emission region of $270 \mathrm{AU}$ (less than 0.02 mas) in size is possible (Wittkowski et al. 1998).

A more speculative suggestion is that a hot and thick accretion flow close to the black hole exists in NGC 1068. It can be described (Beckert \& Duschl 2002) as an advection-dominated flow (ADAF) with a high radiation efficiency $(\epsilon \sim 0.5)$. In such a scenario, synchrotron seed photons are upscattered to produce an IR to X-ray continuum with spectral shape $F_{v} \propto v^{-0.6}$. Assuming that most of the central luminosity of $2 \times 10^{11} L_{\odot}$ (Pier et al. 1994) originates in this hot, innermost region of the accretion flow, where the spectral luminosity $v L_{v}$ peaks at about $10^{19.5} \mathrm{~Hz}$, we can give an upper limit to the $K$-band flux of $F_{v} \lesssim 300 \mathrm{mJy}$ from this flow alone.

The contribution of the central engine to the observed $K$-band flux is relevant only for low optical depth through the dusty torus. For a clumpy distribution of dust clouds there is always a small chance of low optical depth along the particular line of sight to the central emission region. This is consistent with the possible detection of a broad $\mathrm{Br} \gamma$ line by Gratadour et al. (2003) and their modeling of the observed spectral slope in the $K$ band leading to a rather low extinction of $\tau_{K}=1.25$. Therefore, any nuclear emission in excess of the expected flux from the accretion disk can make a significant contribution to the measured $H$ - and $K$-band core flux.

\section{Conclusions}

We observed two $K^{\prime}$-band components in the nuclear region of NGC 1068, a compact core and extended components. The compact $18 \times 39$ mas $(1.3 \times 2.8 \mathrm{pc})$ core shows a tail-shaped, north-western structure at $\mathrm{PA} \sim-16^{\circ}$ and a fainter, southeastern compact component. The north-western extension of the compact core is also visible in the elongated image in Wittkowski et al. (1998), but the $K^{\prime}$-band image reported in the present paper has a much higher SNR, and the new $H$-band image is the first diffraction-limited $H$ image of the compact core.

The PA of approximately $-16^{\circ}$ of the compact $18 \times 39$ mas or $1.3 \times 2.8 \mathrm{pc}$ core is very similar to the position angle of the western wall of the ionization cone. This suggests that the emission from the compact core is both thermal emission and scattered light from dust near the western wall of a 
low-density, conical cavity or from the innermost region of a parsec-scale dusty torus. Dust emission from the corresponding eastern cavity wall is not visible, most likely due to an asymmetric dust distribution. Such an asymmetry is also observed in the MIR.

Dust located between the BLR and NLR can emit and scatter $K^{\prime}$-band radiation. Efstathiou \& Rowan-Robinson (1995) have shown that thermal emission and scattering at such a dust component can explain a $K^{\prime}$-band flux of $350 \mathrm{mJy}$. Torus models which can explain the observed infrared SED by emission solely from the torus (i.e., without dust within the ionization cone) were presented by Granato \& Danese (1994). A simulated $2.2 \mu \mathrm{m}$ image (Granato et al. 1997) shows an elongated shape with a size roughly in agreement with our images. Another possibility is that a large fraction of the observed flux from the compact core is direct thermal radiation from the inner accretion disk or non-thermal direct radiation from the central continuum source, which escapes through regions of moderate extinction.

The extended northern 400 mas component has a PA of approximately $0^{\circ}$. A weaker, south-eastern extended component is visible as well. This north-south structure is in good agreement with the AO images presented by Rouan et al. (1998). The $K^{\prime}$ - and $H$-band fluxes of the compact core were determined to be $350 \mathrm{mJy}$ and $70 \mathrm{mJy}$, respectively.

The northern extended 400 mas structure lies near the western wall of the ionization cone and coincides with the inner radio jet $\left(\mathrm{PA} \sim 11^{\circ}\right)$. We interpret the extended component as radiation scattered by electrons in the NLR and by dust grains near the western wall of the NLR cone. Other possible interpretations of the extended component include thermal emission of dust heated by dynamical interaction with the radio jet and small dust grains transiently heated to high temperatures by single UV photons (Weinberger et al. 1999).

Acknowledgements. Based on observations made with the NASA/ESA Hubble Space Telescope, obtained from the data archive at the Space Telescope Science Institute. STScI is operated by the Association of Universities for Research in Astronomy, Inc. under NASA contract NAS 5-26555.

A.B.M. acknowledges support from the Natural Sciences and Engineering Research Council of Canada (NSERC).

We thank the referee for valuable comments.

\section{References}

Antonucci, R. R. J., \& Miller, J. S. 1985, ApJ, 297, 621

Barvainis, R. 1987, ApJ, 320, 537

Beckert, T., \& Duschl, W. J. 1997, A\&A, 328, 95

Beckert, T., \& Duschl, W. J. 2002, A\&A, 387, 422

Beckert, T., \& Duschl, W. J. 2004, A\&A, submitted

Beckert, T., Duschl, W. J., Mezger, P. G., \& Zylka, R. 1996, A\&A, 307,450

Bland-Hawthorn, J., Gallimore, J. F., Tacconi, L. T., et al. 1997, Ap\&SS, 248, 9

Bock, J. J., Marsh, K. A., Ressler, M. E., \& Werner, M. W. 1998, ApJ, 504, L5
Bock, J. J., Neugebauer, G., Matthews, K., et al. 2000, AJ, 120, 2904

Braatz, J. A., Wilson, A. S., Gezari, D. Y., Varosi, F., \& Beichmann, C. A. 1993, ApJ, 409, L5

Cameron, M., Storey, J. W. V., Rotaciuc, V., et al. 1993, ApJ, 419, 136

Capetti, A., Macchetto, F., Axon, D. J., Sparks, W. B., \& Boksenberg, A. 1995, ApJ, 452, L87

Chelli, A., Perrier, C., Cruz-Gonzalez, I., \& Carrasco, L. 1987, A\&A, 177,51

Christou, J. C., Cheng, A. Y. S., Hege, E. K., \& Roddier, C. 1985, AJ, 90, 2644

Dopita, M. A., Heisler, C., Lumsden, S., \& Bailey, J. 1998, ApJ, 498, 570

Duschl, W. J., Strittmatter, P. A., \& Biermann, P. L. 2000, A\&A, 357, 1123

Efstathiou, A., \& Rowan-Robinson, M. 1995, MNRAS, 273, 649

Efstathiou, A., Hough, J. H., \& Young, S. 1995, MNRAS, 277, 1134

Galliano, E., Alloin, D., Granato, G. L., \& Villar-Martín, M. 2003, A\&A, 412, 615

Gallimore, J. F., Baum, S. A., O’Dea, C. P., \& Pedlar, A. 1996a, ApJ, 458,136

Gallimore, J. F., Baum, S. A., O'Dea, C. P., \& Pedlar, A. 1996b, ApJ, 464, 198

Gallimore, J. F., Baum, S. A., O’Dea, C. P., Brinks, E., \& Pedlar, A. 1996c, ApJ, 462, 740

Gallimore, J. F., Baum, S. A., O’Dea, C. P., \& Pedlar, A. 1997, Nature, 388,852

Glass, I. S. 1997, Ap\&SS, 248, 191

Granato, G. L., \& Danese, L. 1994, MNRAS, 268, 235

Granato, G. L., Danese, L., \& Franceschini, A. 1997, ApJ, 486, 147

Gratadour, D., Clénet, Y., Rouan, D., Lai, O., \& Forveille, T. 2003, A\&A, 411, 335

Greenhill, L. J., Gwinn, C. R., Antonucci, R., \& Barvainis, R. 1996, ApJ, 472, L21

Hofmann, K.-H., \& Weigelt, G. 1986, A\&A, 167, L15

Huré, J.-M. 2002, A\&A, 395, L21

Kishimoto, M. 1999, ApJ, 518, 676

Krolik, J. H., \& Begelmann, M. C. 1988, ApJ, 329, 702

Labeyrie, A. 1970, A\&A, 6, 85

Lohmann, A. W., Weigelt, G., \& Wirnitzer, B. 1983, Appl. Opt., 22, 4028

Macchetto, F., Capetti, A., Sparks, W. B., Axon, D. J., \& Boksenberg, A. 1994, ApJ, 435, L15

Maiolino, R., Marconi, A., \& Oliva, E. 2001, A\&A, 365, 37

Manske, V., Henning, T., \& Men'shchikov, A. B. 1998, A\&A, 331, 52

Marco, O., \& Alloin, D. 2000, A\&A, 353, 465

Marco, O., Alloin, D., \& Beuzit, J. L. 1997, A\&A, 320, 399

McCarthy, D. W., Low, F. J., Kleinmann, S. G., \& Gillet, F. C. 1982, ApJ, 257, L7

Miller, J. S., Goodrich, R. W., \& Mathews, W. G. 1991, ApJ, 378, 47

Muxlow, T. W. B., Pedlar, A., Holloway, A. J., Gallimore, J. F., \& Antonucci, R. R. J. 1996, MNRAS, 278, 854

Nenkova, M., Ivezić, Ž., \& Elitzur, M. 2002, ApJ, 570, L9

Pehlemann, E., Hofmann, K.-H., \& Weigelt, G. 1992, A\&A, 256, 701

Phinney, E. S. 1989, in Theory of Accretion Disks, ed. W. Duschl, F. Meyer, \& J. Frank (Dordrecht: Kluwer), 457

Pier, E. A., \& Krolik, J. H. 1992, ApJ, 401, 99

Pier, E. A., \& Krolik, J. H. 1993, ApJ, 418, 673

Pier, E. A., Antonucci, R., Hurt, T., Kriss, G., \& Krolik, J. 1994, ApJ, 428,124 
Pringle, J. E. 1996, MNRAS, 281, 357

Pringle, J. E. 1997, MNRAS, 292, 136

Puget, J. L., Leger, A., \& Boulanger, F. 1985, A\&A, 142, L19

Reuter, H. P., \& Lesch, H. 1996, A\&A, 310, L5

Rieke, G. H., \& Low, F. J. 1975, ApJ, 199, L13

Rouan, D., Rigaut, F., Alloin, D., et al. 1998, A\&A, 339, 687

Rouan, D., Lacombe, F., Gendron, E., et al. 2004, A\&A, 417, L1

Sanders, D. B., Phinney, E. S., Neugebauer, G., Soifer, B. T., \& Matthews, K. 1989, ApJ, 347, 29

Scoville, N. Z., Matthews, K., Carico D. P., \& Sanders D. B. 1988, ApJ, 327, L61
Thatte, N., Quirrenbach, A., Genzel, R., Maiolino, R., \& Tecza, M. 1997, ApJ, 490, 238

Tully, R. B. 1988, Nearby Galaxies Catalog (Cambridge University Press)

Vollmer, B., Beckert, T., \& Duschl, W. J. 2004, A\&A, 413, 949

Weigelt, G., \& Wirnitzer, B. 1983, Opt. Lett., 8, 389

Weigelt, G. P. 1977, Opt. Commun., 21, 55

Weinberger, A. J., Neugebauer, G., \& Matthews, K. 1999, AJ, 117, 2748

Wittkowski, M., Balega, Y., Beckert, T., et al. 1998, A\&A, 329, L45 (Paper I) 